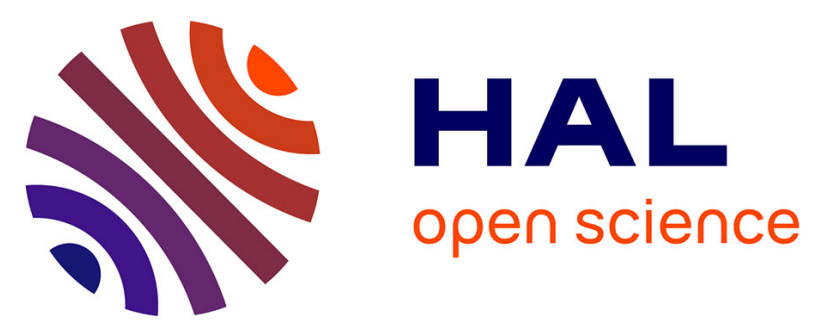

\title{
Properties of helium bubbles in covalent systems at the nanoscale: A combined numerical and experimental study
}

J. Dérès, M.-L. David, K. Alix, C. Hébert, D. T L Alexander, Laurent Pizzagalli

\section{To cite this version:}

J. Dérès, M.-L. David, K. Alix, C. Hébert, D. T L Alexander, et al.. Properties of helium bubbles in covalent systems at the nanoscale: A combined numerical and experimental study. Physical Review B: Condensed Matter and Materials Physics (1998-2015), 2017, 96 (1), 10.1103/PhysRevB.96.014110 . hal-02114780

\section{HAL Id: hal-02114780 \\ https://hal.science/hal-02114780}

Submitted on 29 Apr 2019

HAL is a multi-disciplinary open access archive for the deposit and dissemination of scientific research documents, whether they are published or not. The documents may come from teaching and research institutions in France or abroad, or from public or private research centers.
L'archive ouverte pluridisciplinaire HAL, est destinée au dépôt et à la diffusion de documents scientifiques de niveau recherche, publiés ou non, émanant des établissements d'enseignement et de recherche français ou étrangers, des laboratoires publics ou privés. 


\title{
Properties of helium bubbles in covalent systems at the nanoscale: A combined numerical and experimental study
}

\author{
J. Dérès, ${ }^{1, *}$ M.-L. David, ${ }^{1}$ K. Alix,${ }^{1}$ C. Hébert, ${ }^{2}$ D. T. L. Alexander, ${ }^{2}$ and L. Pizzagalli ${ }^{1}$ \\ ${ }^{1}$ Departement of Physics and Mechanics of Materials, Institut P', CNRS-Université de Poitiers UPR 3346, SP2MI, BP 30179, \\ F-86962 Futuroscope Chasseneuil Cedex, France \\ ${ }^{2}$ CIME, EPFL-SB-CIME-GE, CH-1015 Lausanne, Switzerland
}

(Received 20 March 2017; revised manuscript received 31 May 2017; published 17 July 2017)

\begin{abstract}
The properties of nanometric-sized helium bubbles in silicon have been investigated using both spatially resolved electron-energy-loss spectroscopy combined with a recently developed method, and molecular-dynamics simulations. The experiments allowed for an accurate determination of size, aspect ratio, and helium density for a large number of single bubbles, whose diameters ranged from 6 to $20 \mathrm{~nm}$. Very high helium densities, from 60 to $180 \mathrm{He} \mathrm{nm}^{-3}$, have been measured depending on the conditions, in stark contrast with previous investigations of helium bubbles in metal with similar sizes. To supplement experiments on a smaller scale, and to obtain insights into the silicon matrix state, atomistic calculations have been performed for helium bubbles in the diameter range 1-13 nm. Molecular-dynamics simulations revealed that the maximum attainable helium density is critically related to the strength of the silicon matrix, which tends to yield by amorphization at the highest density levels. Calculations give helium density values for isolated single bubbles that are typically lower than measurements. However, excellent agreement is recovered when the interactions between bubbles and the presence of helium interstitials in the matrix are taken into account. Both experiments and numerical simulations suggest that the Laplace-Young law cannot be used to predict helium density in nanometric-sized bubbles in a covalent material such as silicon.
\end{abstract}

DOI: 10.1103/PhysRevB.96.014110

\section{INTRODUCTION}

Noble gas species (helium, neon, etc.) are characterized by filled electronic shells, leading to a quasi absence of chemical reactivity. As a consequence, they exhibit unique behaviors when interacting with diverse materials [1]. Hence, they are used for processing semiconductors, yielding an inert atmosphere during growth, ion beam milling of surfaces, or plasma etching. Light noble gas species such as helium or neon are also potentially useful in the gettering process for electronic devices [2], to relax strain in pseudomorphic $\mathrm{SiGe/Si} \mathrm{heterostructures} \mathrm{[3],} \mathrm{and} \mathrm{for} \mathrm{the} \mathrm{production} \mathrm{of} \mathrm{silicon-}$ on-insulator wafer substrates using the smartcut process [4]. In an astrophysical context, noble gas atoms have been found as impurities in interstellar dusts [5], and they can be used as markers in models attempting to describe stars evolution. Finally, there are also extensive investigations in the context of nuclear applications, since helium is produced in large quantities coming from neutron-induced transmutation reactions. In fusion reactors, the plasma also generates a high helium flux interacting with the wall. Helium accumulation into structural, confining, or fuel cladding materials leads to the formation of extended defects like bubbles [1,6], potentially resulting in swelling, embrittlement, surface roughening, and blistering, all mechanisms that can dramatically degrade the mechanical properties.

The driving force leading to extended defects formation is the nonsolubility of noble gas species due to their chemical inertness. Noble gas atoms tend to aggregate to lower the energy cost, occupying available space at defects or generating some if possible. As a result, the formation of gas-filled

\footnotetext{
*julien.deres@univ-poitiers.fr
}

extended defects such as platelets and bubbles is generally favored. This phenomenon is essentially independent of the host materials, since it has been reported in metals [6-10], covalent systems such as silicon [11-20], silicon carbide [21-26], gallium nitride [27], and disordered materials [2830]. However, knowing why the bubbles form does not provide information about the formation mechanism itself, or about the properties of these bubbles. A key property is the gas density contained in these bubbles, which is directly related to the internal gas pressure through an appropriate equation of state. It is a required parameter in available models attempting to describe the formation and evolution of these defects [31,32].

The earliest attempts at measuring the gas density in bubbles were often indirect (by measuring the material's volumic expansion, for instance), and they assumed that all available noble gas atoms were located in the bubbles [7]. Still, the fact that these estimations often lead to unrealistically huge gas density in bubbles casts some doubt about the validity of this assumption. Other measurements were direct $[8,18,33,34]$, but they provided data averaged over a large quantity of bubbles, which were then assumed to share the same characteristics. More recently, spatially resolved electron-energy-loss spectroscopy experiments performed in a transmission electron microscope allowed the direct probing of helium density in a single bubble [35-40]. In each of these studies, a few bubbles were characterized, with values in the range $15-76 \mathrm{He} \mathrm{nm}^{-3}$ in metals [35,36], in nanoporous amorphous silicon [40], and in minerals [30], and in the range $60-173 \mathrm{He} \mathrm{nm}^{-3}$ in crystalline silicon [37-39]. Using available equations of state [41,42], these values correspond to internal pressures of 0.1-2.0 GPa in the first case and 1-21 GPa in the second one.

There is a broad consensus in the scientific community that the internal pressure in gas-filled bubbles at equilibrium can 
also be directly obtained from the well-known Laplace-Young law $\Delta P=2 \gamma / r$, with $\gamma$ the surface tension, $r$ the bubble radius, and $\Delta P$ the pressure difference between both sides of the bubble-matrix interface. An important consequence of this equation is that the smaller the bubble, the higher the gas density. This seems to be verified for helium bubbles in steel [36], and to a lesser extent in a PdPt alloy [35]. However, such a dependence is not found for covalent materials [37-39]. Since this may simply have been due to the very small number of bubbles investigated so far, interpretation is indefinite, a limitation that we address here with greater statistical sampling. The Laplace-Young law is also commonly used to estimate the order of magnitude of the internal pressure of gas-filled bubbles. However, comparing predictions with the above-mentioned measurements reveals a large discrepancy, which suggests that either the Laplace-Young law is not appropriate, or that the helium-filled bubbles are often not at equilibrium. Note that in the literature, it is usually assumed that the surface tension is equal to the surface energy, and that the pressure transmitted to the matrix is negligible. The latter assumption is clearly not verified when local deformation is observed in the materials due to the pressure exerted by the bubbles.

Another approach for determining the internal noble gas density could be atomistic simulations. Those have been performed for helium bubbles in various host materials [43-53]. Nevertheless, in almost all these studies, the helium density is not an outcome of the calculation, rather it is set as an initial parameter. These numerical simulations are then valuable tools to reveal various properties of the bubble and of the host material for a given internal gas density, but they cannot be used to directly determine this density.

These different points suggest that we clearly need additional experimental information regarding the internal density of gas-filled bubbles, especially in the case of helium bubbles in silicon, for which an unusual behavior has been reported $[37,38]$. We have recently developed a spatially resolved electron-energy-loss spectroscopy (EELS) approach based on energy-filtered transmission-electron-microscopy-spectrumimaging acquisition of the data. This technique both allows an investigation of a large number of bubbles [39], and avoids desorption under the electron beam [37]. The results of these experiments are reported in this paper. The helium density has been measured in more than 100 bubbles, with diameters ranging from 5 to $20 \mathrm{~nm}$. This unprecedented statistic reveals trends in plain disagreement with the Laplace-Young relation, since larger bubbles exhibit similar or slightly larger helium densities than smaller ones. These densities are also typically one order of magnitude higher than predicted values. In addition, largescale atomistic simulations have been performed, modeling bubbles with dimensions and helium densities comparable to experiments. These calculations allowed for the determination of several properties that are difficult to access in experiments, such as the detailed structure of the bubble interface and of the silicon matrix. They also revealed that the measured helium density values correspond to bubbles close to the mechanical stability limit.

This paper is organized in five parts. First, experimental conditions and results are detailed. Then, simulation conditions and results are reported. Finally, the results are discussed.

\section{EXPERIMENTAL DETAILS}

Helium bubbles were synthesized in monocrystalline $p$ type $\mathrm{Si}$ wafers using $50 \mathrm{keV}$ helium ions at a fluence of $7.5 \times 10^{16}$ at $\mathrm{cm}^{-2}$. The implantations were performed at room temperature with a current density of $0.01 \mathrm{~A} / \mathrm{m}^{2}$. Within these experimental conditions, SRIM software calculations [54] predict a mean projected range of the ions of $410 \mathrm{~nm}$ with respect to the free surface and a straggling of $110 \mathrm{~nm}$. The samples were then annealed at temperatures of 500 or $700{ }^{\circ} \mathrm{C}$ in a tubular furnace under vacuum. These implantation conditions were chosen to create a condensed system of bubbles of nanometric size with the annealing conditions tuned to obtain different morphologies and sizes of bubbles [39].

The bubbles were next studied using spatially resolved EELS. For that purpose, cross-sectional transmission electron microscopy (TEM) samples were prepared by mechanical polishing down to $10 \mu \mathrm{m}$ thick and then ion milling in a GATAN-PIPS apparatus at low energy (2.5 keV Ar) and low incidence $\left( \pm 8^{\circ}\right)$. To minimize irradiation damage, a final step was performed at $\pm 4^{\circ}$ for $2 \mathrm{~min}$.

The spatially resolved EELS experiments were performed using a JEOL JEM 2200FS microscope fitted with an omega filter and a Gatan Ultrascan $2048 \times 2048$ pixels CCD camera. The data were recorded and analyzed using the energy-filtered transmission electron microscopy spectrum image (EFTEMSI) approach that we recently developed. The datacubes were acquired with a collection angle of $5.65 \mathrm{mrad}, 0.2 \mathrm{eV}$ energy steps between energy planes, a slit of $1 \mathrm{eV}$, and between -3 and $32 \mathrm{eV}$ in two steps. The elastic peak was first acquired with an $0.5 \mathrm{~s}$ exposure time, and the second part of the datacube [10-32] eV was recorded with a 5 s exposure time.

The combined correction of the nonisochromaticity and spatial drift was realized using a home-made procedure. The He $K$-edge was extracted after median filtering, multiple scattering deconvolution, and a fit of the $\mathrm{SiO}_{2} / \mathrm{Si}$ interface plasmon, Si plasmon, and cavity plasmon by Gaussian or pseudo-Voigt functions. The acquisition and treatment procedures of the spectrum images are described in further detail in Ref. [39]. The helium density, $n_{\mathrm{He}}$, is determined through its relation with the blueshift of the He $K$-edge, $\Delta E$, using [37,55,56]

$$
n_{\mathrm{He}}=\frac{1}{C_{\mathrm{He}}} \Delta E,
$$

where $\Delta E=E-E_{0} . E$ is the energy of the He $K$-edge measured in the bubble under study. $E_{0}$ is the energy of the He $K$-edge for the free atom, which is taken at $21.75 \mathrm{eV}$ in this study and corresponds to the energy position of the He $K$-edge for nearly empty bubbles. To determine this value, in situ isothermal annealing experiments in TEM, to be published elsewhere, were carried out on similar systems of bubbles. Moreover, $C_{\mathrm{He}}$ in Eq. (1) is equal to $0.015 \mathrm{eV} \mathrm{nm}^{3}$ as determined by previous experiments in similar systems [37].

Prior to any EFTEM-SI acquisition, underfocused $(-1000 \mathrm{~nm})$ zero-loss energy-filtered images were acquired. Quantitative analyses of these images were performed to retrieve the aspect ratio and size of bubbles. The method used is based on a standard image thresholding procedure, which has proven to be very successful to analyze embedded nanoparticles [57]. However, it results in an error on the 


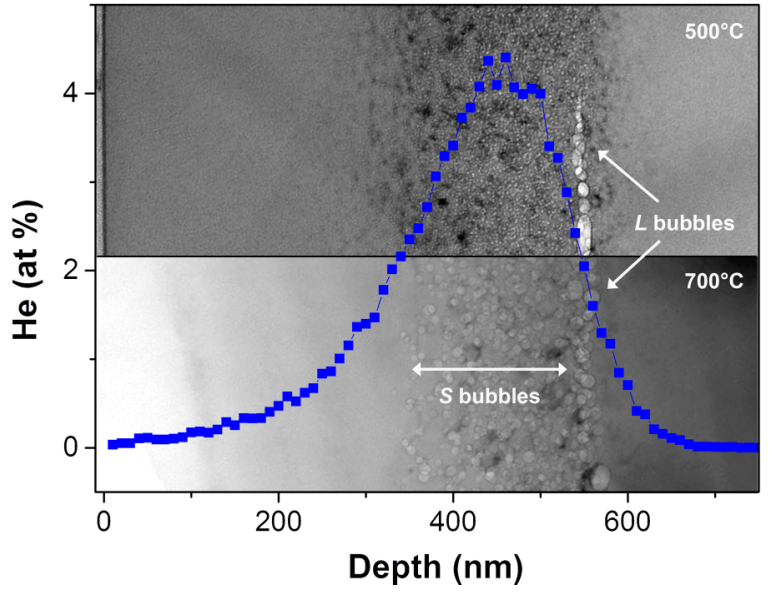

FIG. 1. Zero loss energy-filtered TEM micrographs (underfocus, $5 \mathrm{eV} \mathrm{slit})$ of Si samples implanted with helium $\left(7.5 \times 10^{16} \mathrm{He} \mathrm{cm}^{-2}\right.$, $50 \mathrm{keV}$ ) and annealed at $500^{\circ} \mathrm{C}$ (upper part) and $700{ }^{\circ} \mathrm{C}$ (lower part) together with the helium concentration (blue squares) profile as determined by SRIM. For each micrograph, the contrast has been adjusted.

bubble size that is due to the thresholding process and to the thickness of the Fresnel fringe used to image the bubbles. Size measurements on both energy-filtered images on the silicon plasmon and underfocused bright-field images were performed and compared for several bubbles, yielding an error of $\pm 1 \mathrm{~nm}$. The bubble aspect ratio is defined as $a / b$, with $a$ the largest dimension. In the following, the bubble diameter is defined as the diameter of a disk having the same area as an ellipse of parameters $a$ and $b$.

\section{EXPERIMENTAL RESULTS}

The microstructure of the samples is shown in Fig. 1 together with the helium concentration profile as calculated by SRIM [54]. As seen, the chosen implantation and annealing conditions lead to the formation of a layer of bubbles centered around the helium profile, i.e., located between about 320 and $550 \mathrm{~nm}$ away from the sample surface. In both cases, the bubbles with the largest diameters are located at the deeper edge of the bubble layer. In the following, these bubbles are referred to as $L$ (large) whereas the smaller bubbles, homogeneously distributed in the bubble band, are referred to as $S$ (small) (see Fig. 1).

For each sample, two TEM thin foils were prepared and several zones analyzed. Typical examples of explored zones are shown in Figs. 2 and 3 for the 500 and $700{ }^{\circ} \mathrm{C}$ annealed samples, respectively. Comparing the underfocus micrographs [Figs. 2(a) and 3(a)] with the corresponding He chemical maps extracted from the EFTEM-SI [Figs. 2(b) and 3(b)] reveals that some of these bubbles are empty and that superposition of bubbles may occur. In these systems, the main limitation to ensure a correct quantitative analysis of numerous individual bubbles is the thickness of the sample as compared to the bubble diameter. As reported in Ref. [39], there is an optimal thickness of the sample, i.e., thin enough so that the bubbles are not superimposed and thick enough so that there is a maximum of full bubbles to be analyzed (the other ones may have been
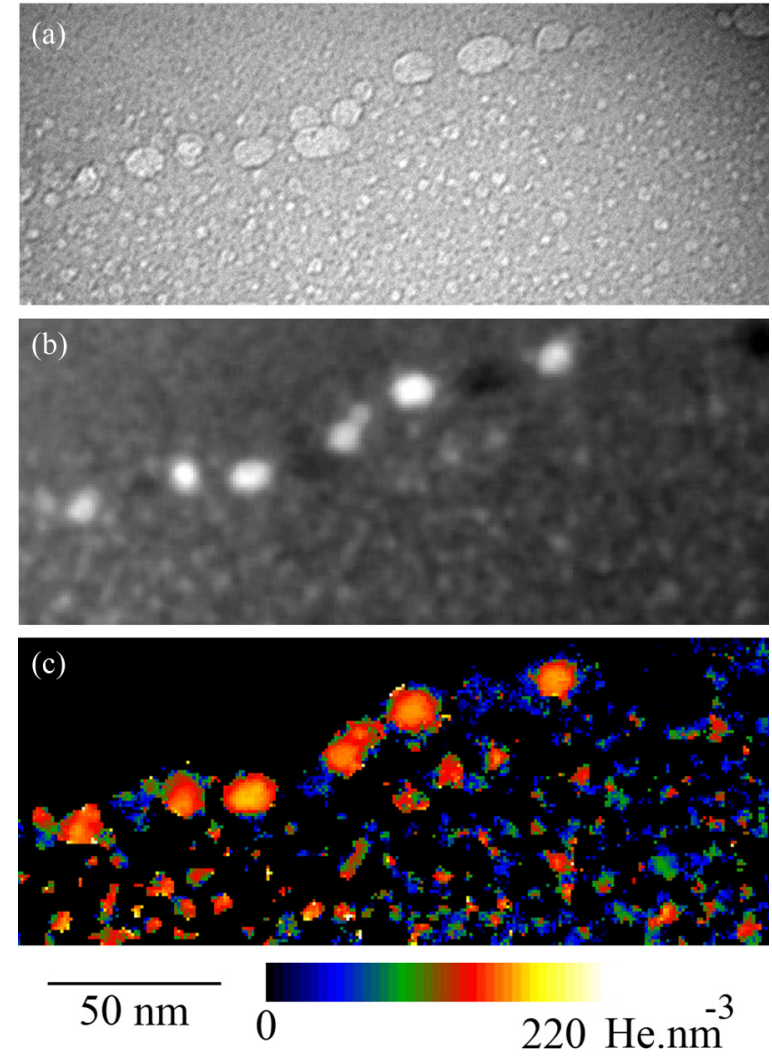

FIG. 2. (a) Zero loss energy-filtered micrograph ( $1 \mathrm{eV}$ slit) in underfocus conditions $(-1000 \mathrm{~nm})$ of a $7.5 \times 10^{16} \mathrm{He} \mathrm{cm}^{-2}, 50 \mathrm{keV}$ implanted silicon sample annealed at $500{ }^{\circ} \mathrm{C}, 1 \mathrm{~h}$. (b) Energy-filtered image on the He $K$-edge $(24.2 \pm 1 \mathrm{eV})$ extracted from a spectrum image acquired on the same region as (a). (c) Corresponding helium density map.

cut during the sample preparation). This is a serious limitation. In particular, for the $500{ }^{\circ} \mathrm{C}$ annealed sample, only two of the datacubes we recorded meet these criteria, leading to less analyzable data than for the $700{ }^{\circ} \mathrm{C}$ annealed sample.

The corresponding helium density maps determined using our procedure are shown in Figs. 2(c) and 3(c). In the case of the $500^{\circ} \mathrm{C}$ annealed sample, helium can be detected in the $S$ bubbles, but their diameter is too small (3-4 nm) to ensure an accurate helium density quantification. Therefore, the analysis is restricted to the $L$ bubbles for this sample.

The quantities extracted from Figs. 2(a) and 2(c) are reported in Fig. 4. The $L$ bubbles exhibit a diameter ranging from 7 to $16 \mathrm{~nm}$ (11 nm in average) and their aspect ratio is between 1 and 1.9 (1.3 in average). Figure 4(a) clearly indicates that the aspect ratio increases with the diameter of the bubbles, i.e., the largest bubbles are less spherical than the smaller ones. The helium density measured in these bubbles is characterized by a broad distribution, with values ranging from 120 to $180 \mathrm{He} \mathrm{nm}^{-3}$. An increase of the helium density as a function of the diameter and of the aspect ratio is suggested in Fig. 4, although three of the data points clearly do not follow this trend. This behavior has to be confirmed with a higher statistic.

Annealing at $700{ }^{\circ} \mathrm{C}$ leads to larger and more spherical bubbles (Fig. 5). However, the two $S$ and $L$ bubble sets can still be distinguished. The $S$ bubbles exhibit a diameter ranging 

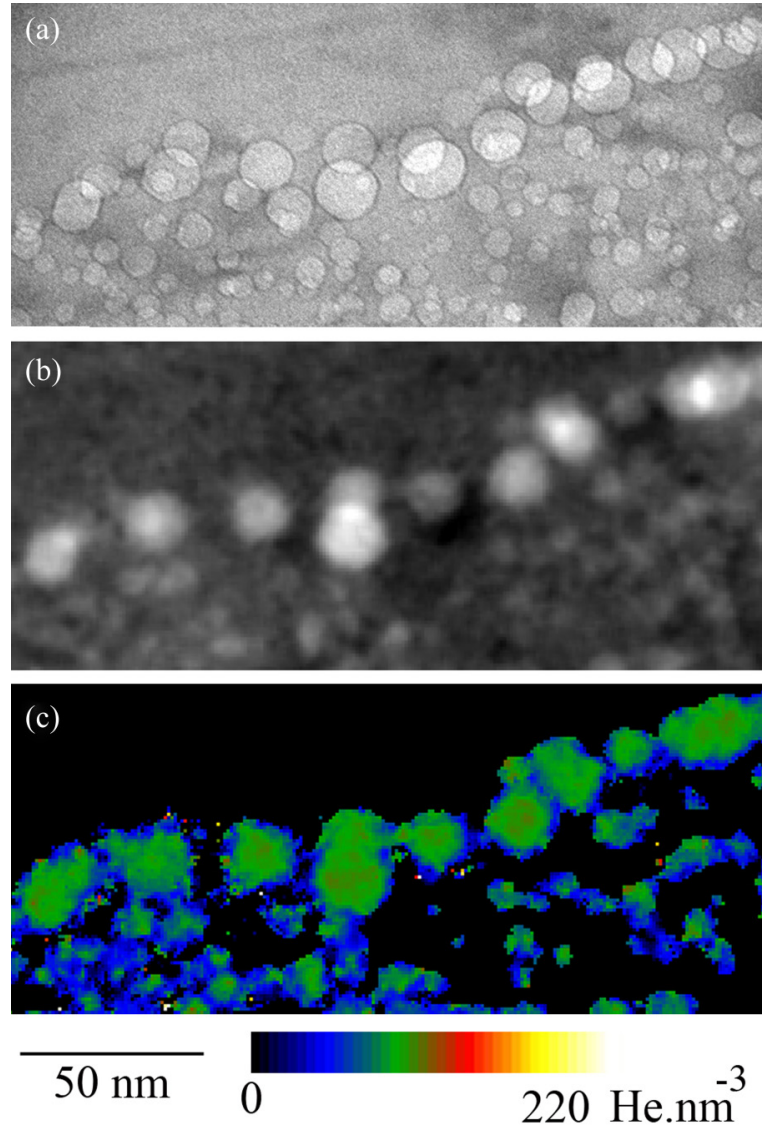

FIG. 3. (a) Zero loss energy-filtered micrograph (1 eV slit) in underfocus conditions $(-1000 \mathrm{~nm})$ of a $7.5 \times 10^{16} \mathrm{He} \mathrm{cm}^{-2}, 50 \mathrm{keV}$ implanted silicon sample annealed at $700{ }^{\circ} \mathrm{C}, 1 \mathrm{~h}$. (b) Energy-filtered image on the He $K$-edge $(23.4 \pm 1 \mathrm{eV})$ extracted from a spectrum image acquired on the same region as (a). (c) Corresponding helium density map.

from 6 to $12 \mathrm{~nm}$ (average $9 \mathrm{~nm}$ ), whereas the $L$ bubble diameter ranges from 12 to $20 \mathrm{~nm}$ (average $16 \mathrm{~nm}$ ). Both sets are characterized by a rather spherical shape with an aspect ratio from 1 to 1.3 [Fig. 5(a)]. As seen in Fig. 5(b), the helium density in both types of bubbles is much lower than that determined in the bubbles formed after an annealing at $500{ }^{\circ} \mathrm{C}$ : the helium density in the $L$ bubbles varies between 60 and $90 \mathrm{He} \mathrm{nm}^{-3}$, whereas it can even be smaller in the $S$ bubbles $\left(40 \mathrm{He} \mathrm{nm}^{-3}\right)$. These data suggest that, whatever the bubble diameter, there is a maximum helium density of about $90 \mathrm{He} \mathrm{nm}^{-3}$ that can be reached for the $700^{\circ} \mathrm{C}$ annealing. Moreover, it is clearly seen that bubbles of similar diameter can exhibit different helium densities, whereas similar helium densities can be found in bubbles of various diameters.

Finally, it is noteworthy that all these results are extracted from several datacubes recorded in different zones (represented in different colors in Figs. 4 and 5). Taking into account the experimental uncertainties, the results did not show any dependence on the datacube, indicating the robustness of our acquisition and treatment procedure.

\section{SIMULATION DETAILS}

Our model first includes a cubic silicon supercell, periodically repeated in three dimensions. To build a helium-filled bubble, a void was first created by removing silicon atoms contained in a sphere of diameter $d_{0}$ in the center of the box. Helium atoms were next inserted into the cavity. The initial helium density is expressed in terms of the $\mathrm{He} / V$ ratio, the number of helium atoms divided by the number of removed silicon atoms (vacancies). As will become clear in the following, ratios between 1 and 6 are relevant in our study, and investigations will be carried out within this range. We have considered different initial bubble diameters, $d_{0}$, ranging from 1 to $13 \mathrm{~nm}$. To investigate helium bubbles in different conditions, various supercell sizes were used. The largest one contains about 6 million atoms in $(48.9 \mathrm{~nm})^{3}$, and it allows for study of a quasi-isolated bubble. Decreasing the size increases the interaction between bubbles in periodic replicas. The smallest supercell dimension considered in this work is $(9.8 \mathrm{~nm})^{3}$, and it allows for modeling a network of strongly interacting helium bubbles.

We have carried out molecular-dynamics (MD) simulations using the LAMMPS code [58] with a 1 fs time step. The modified embedded-atom-method (MEAM) formalism was used to describe the interatomic interactions [59], with the parameters reported in Ref. [51]. The $\mathrm{Si}-\mathrm{Si}$ interaction is the "Si92" potential [60], yielding a bulk silicon lattice constant $a_{0}$ equal to $5.431 \AA$. The very high helium densities
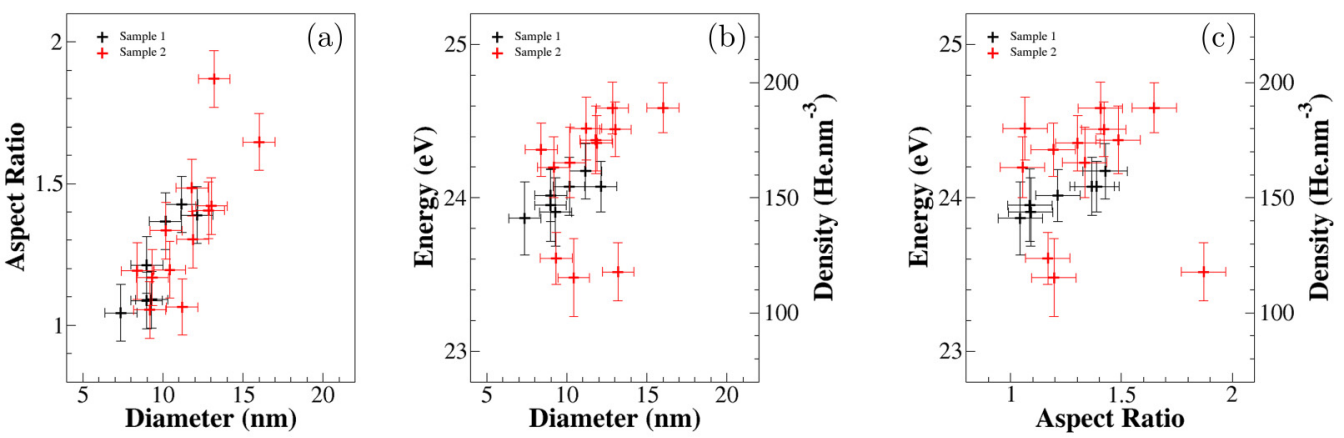

FIG. 4. (a) Aspect ratio as a function of the bubble diameter, (b) helium density as a function of the bubble diameter, and (c) helium density as a function of the aspect ratio for a $7.5 \times 10^{16} \mathrm{He} \mathrm{cm}^{-2}, 50 \mathrm{keV}$ implanted silicon sample annealed at $500{ }^{\circ} \mathrm{C}, 1 \mathrm{~h}$. The spectrum images were recorded on two different TEM thin foils [different colors in (a), (b), and (c)]. Only the $L$ bubbles are analyzed. 

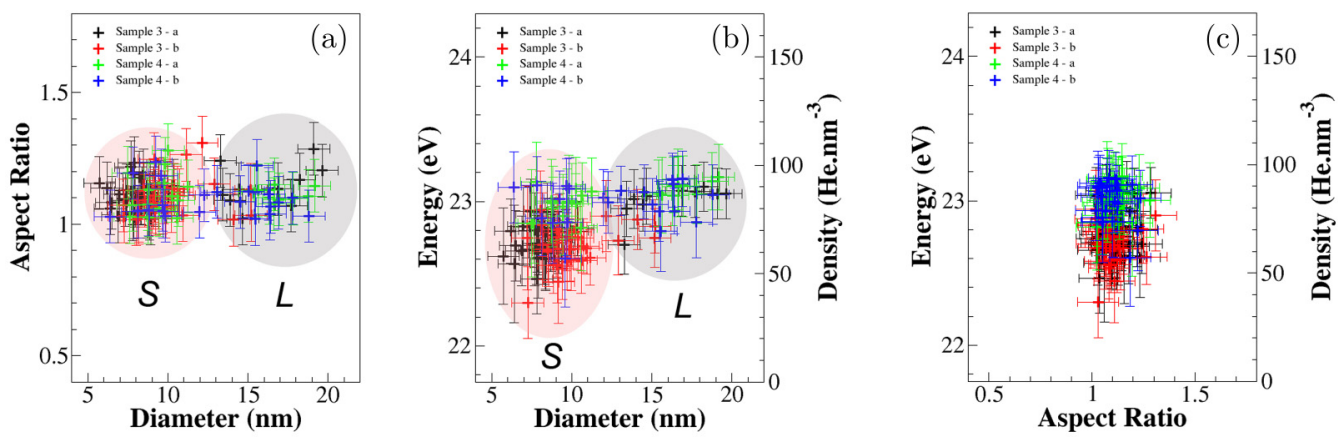

FIG. 5. (a) Aspect ratio as a function of the bubble diameter, (b) helium density as a function of the bubble diameter, and (c) helium density as a function of the aspect ratio for a $7.5 \times 10^{16} \mathrm{He} \mathrm{cm}^{-2}, 50 \mathrm{keV}$ implanted silicon sample annealed at $700{ }^{\circ} \mathrm{C}, 1 \mathrm{~h}$. The spectrum images were recorded on two different regions of two TEM thin foils [different colors in (a), (b), and (c)].

measured in bubbles imply that the pressure transmitted to the silicon matrix is significant. It is then required that the potential can reliably reproduce the mechanical properties of silicon. We computed the elastic constants $C_{11}=167 \mathrm{GPa}$, $C_{12}=63 \mathrm{GPa}$, and $C_{44}=76 \mathrm{GPa}$, in excellent agreement with experiments. It is also found that the potential is able to predict the most stable configuration for a screw dislocation [61]. The Si-He interaction was shown to correctly reproduce the properties of helium atoms in silicon [51], in particular the fact that an interstitial tetrahedral site is energetically more favorable than a substitution site. Concerning the $\mathrm{He}-\mathrm{He}$ MEAM parametrization, the attractive part was fitted on the Tang and Toennies potential [62], while the repulsive one was fitted on first-principles calculations [51]. Preliminary calculations using the potential showed that it predicts no clustering of helium interstitials in silicon, in accordance with first-principles calculations [63].

Two different methods for introducing helium atoms into the cavity were tested. In the first one, helium atoms were inserted into the cavity as a spherical piece of hcp crystal, chosen as a reference structure of solid helium. The hcp lattice parameter was tuned to obtain different initial helium densities. In the second method, helium atoms were randomly inserted. The difference between the two methods on the final result is negligible. In both cases, it is important to avoid a situation in which two atoms are initially very close, causing unrealistically large forces at the beginning of the MD simulation. For the first method, the spherical helium crystal has to be smaller than the created cavity so that the silicon and helium atoms are not too close at the interface. For random insertion, the probability for two atoms to be very close increases with the $\mathrm{He} / V$ ratio. Preliminary MD simulations were thus performed in the $N V E$ ensemble during $0.1 \mathrm{ps,}$ with the condition that the maximum distance an atom can move in one time step is $0.1 \AA$. Forces were then relaxed to obtain an initial configuration with negligible residual forces [64].

In a second step, MD calculations were performed in the $N V T$ ensemble with a Nose-Hoover thermostat set at different temperatures. We considered 80 and $300 \mathrm{~K}$ to compare with experimental measurement temperatures, and 573, 823, and $973 \mathrm{~K}$ to compare with experimental annealing temperatures. MD runs were performed with a duration depending on the temperature: from $40 \mathrm{ps}$ at $80 \mathrm{~K}$ to $120 \mathrm{ps}$ at $973 \mathrm{~K}$ in order to reach a steady state. One important point is that thermal dilation has to be taken into account in order to avoid an additional pressure in the supercell. We have thus calculated the bulk Si equilibrium lattice parameter for each temperature in independent $N P T$ bulk calculations, which was next used for the bubble simulations. The calculated thermal expansion coefficient is $1.4 \times 10^{-5} \mathrm{~K}^{-1}$, which is slightly larger than the experimental value $\left(2.6 \times 10^{-6} \mathrm{~K}^{-1}\right)[65]$. The simulation conditions are summarized in Table I.

Unless specified, the final helium density is calculated by dividing the number of helium atoms in the bubble by the volume of the cavity. The latter is found by summing the Voronoi volumes associated with all helium atoms. The final diameter $d$ is computed from the bubble volume.

\section{MOLECULAR-DYNAMICS CALCULATIONS}

\section{A. Isolated bubble}

We first consider a single isolated bubble embedded in pristine bulk silicon (a representative example is shown in Fig. 6). For an initial helium density $\mathrm{He} / V=1$ [Fig. 6(a)], the number of helium atoms introduced in the bubble is equal to the number of removed silicon atoms. A gap is clearly visible between clustering helium atoms and the silicon matrix. The $\mathrm{Si}-\mathrm{He}$ repulsive interaction being stronger than the $\mathrm{He}-\mathrm{He}$ one, the helium density is not sufficient to compensate for the higher repulsion at the interface. Such a gap has already been shown in the case of a helium bubble in iron $[46,50,66]$. Note that the presence of the gap implies that the bubble volume is slightly underestimated at low helium density. Indeed, in a Voronoi volume decomposition, half of the gap volume is considered belonging to the silicon atoms at the interface. Therefore, the wider the gap, the larger is the volume underestimation.

TABLE I. Time and lattice parameter considered for different temperatures.

\begin{tabular}{lccccc}
\hline \hline$T(\mathrm{~K})$ & 80 & 300 & 573 & 823 & 973 \\
\hline Time (ps) & 40 & 60 & 80 & 100 & 120 \\
$a_{0}(\AA)$ & 5.437 & 5.453 & 5.473 & 5.493 & 5.505 \\
\hline \hline
\end{tabular}



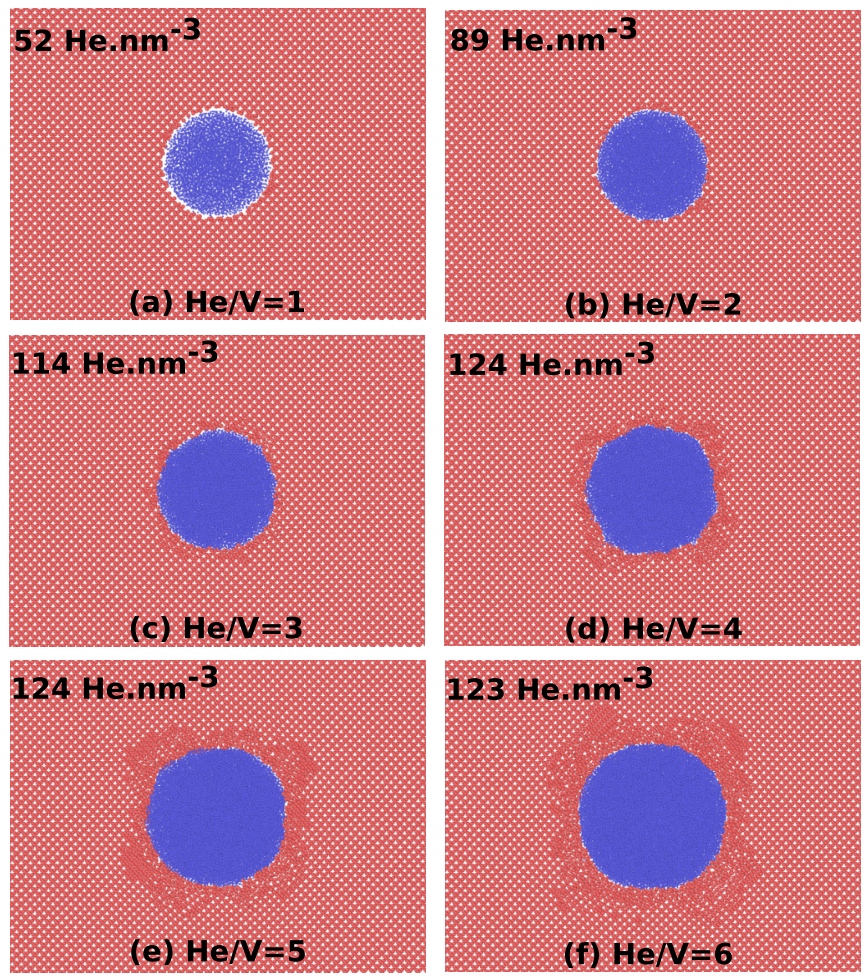

FIG. 6. Cross section of a simulated $d_{0}=6.5 \mathrm{~nm}$ helium bubble at $300 \mathrm{~K}$ for different initial helium densities. Silicon and helium atoms are colored in red and blue, respectively. Only a part of the simulation system is shown for clarity.

At higher initial densities $[\mathrm{He} / V=2-6$, Figs. 6(b)-6(f)], there are enough helium atoms in the bubble to compensate for the $\mathrm{Si}-\mathrm{He}$ repulsion so that the gap disappears. At $\mathrm{He} / V=3$ [Fig. 6(c)], the silicon atoms near the interface seem to be displaced out of their original lattice sites. The volume occupied by the helium atoms clearly increases. At higher initial densities [Figs. 6(d)-6(f)], the apparent deformation of the matrix around the bubble is expanding over a larger region, together with an increase of bubble volume.

This visual analysis suggests that the matrix is plastically deformed in the vicinity of the bubble. We indeed checked that these atomic displacements were irreversible by removing helium atoms from the final state and further relaxing the system. To better characterize the state of the matrix, we performed additional investigations, as reported in the Supplemental Material [67]. Both a local structural analysis and the calculation of radial distribution functions revealed that the silicon matrix yields by amorphization in the vicinity of the bubble interface. The amorphized region grows as a function of the helium density. In addition, a DXA dislocation analysis [68] was performed with the scientific visualization and analysis software OVITO [69], and no dislocations were found.

Figure 7 represents the final helium density and bubble diameter versus the initial helium density for a bubble of initial diameter $d_{0}=6.5 \mathrm{~nm}$ at different temperatures. For $\mathrm{He} / V=1$, the helium density and the diameter of the bubble are close to their initial values. For higher $\mathrm{He} / V$ ratios, the

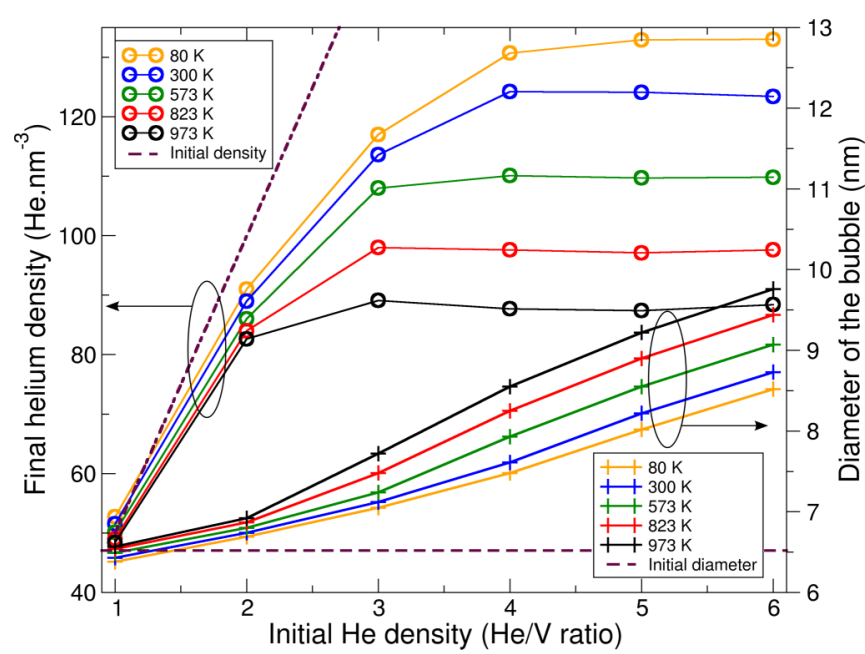

FIG. 7. Final helium density (circle) and diameter of the bubble (crosses) vs the $\mathrm{He} / V$ ratio for a $6.5 \mathrm{~nm}$ initial diameter bubble (in a $(48.9 \mathrm{~nm})^{3}$ supercell). The dashed lines correspond to unrelaxed bubbles. Five temperatures are considered (different colors).

final helium density progressively departs from the unrelaxed initial density until it saturates.

The helium density saturation is therefore obviously related to the bubble volume increase and to the deformation of the matrix (Fig. 7, crosses). In fact, the growing bubble volume compensates for the increase of the initial helium density. The density curves exhibit a clear inflection for a $\mathrm{He} / V$ ratio between 2 and 4 . High temperatures allow for plastic yielding at lower $\mathrm{He} / V$ ratios than at low temperatures, hinting that the plastic deformation of the matrix is thermally activated.

Note that in this work, the simulations were performed by increasing the temperature (in a single run), starting from 80 to $973 \mathrm{~K}$. In some previous theoretical investigations, simulations were apparently made in a single run with decreasing temperatures [46]. In that case, plastic deformations could occur at the beginning of the run due to high temperatures. The calculated densities would thus be underestimated for all temperatures except the highest one (e.g., $973 \mathrm{~K}$ here).

Figure 8 reports the final helium densities in the bubble versus its final diameter. For $\mathrm{He} / V=1$, the helium density decreases slightly, whereas for higher ratios it increases with the diameter of the bubble. The decrease observed for $\mathrm{He} / V=1$ is essentially due to the calculation of the bubble volume. The volume underestimation is more important for small bubbles, leading to an apparent higher density, because the interface over volume ratio is larger. At higher ratios, the helium density increases with the diameter of the bubble.

The values determined experimentally are also reported in Fig. 8. As can be seen, the helium densities measured in the bubbles annealed at $700{ }^{\circ} \mathrm{C}$ are in the range of calculations with $\mathrm{He} / V$ ratios 1 and 2 . Nevertheless, for samples annealed at $500^{\circ} \mathrm{C}$, the measured helium densities are higher than those that can be reached using molecular dynamics. We have thus deepened our investigation and examined parameters that could influence the helium density in the bubble. 


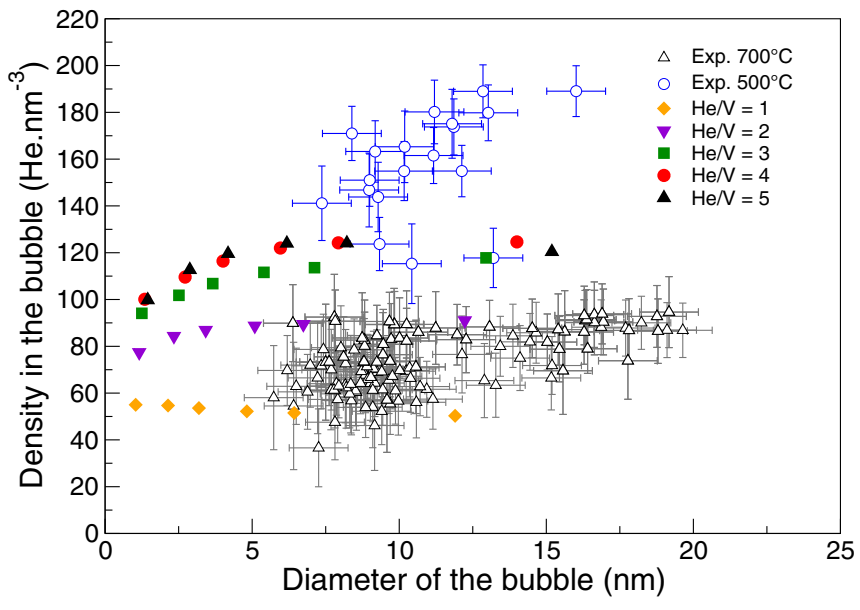

FIG. 8. Helium density in the bubble vs final diameter at $300 \mathrm{~K}$. Comparison between simulations (filled symbols) and experiments for samples annealed at 500 and $700{ }^{\circ} \mathrm{C}$ (empty symbols).

\section{B. Interaction between bubbles}

We first investigate a possible effect of the interaction between bubbles. Indeed, experimentally, one can estimate that the separation between bubbles could be as small as a few nanometers (Fig. 3). Only isolated bubbles were studied in our first simulations, i.e., the interactions between bubbles were negligible. The influence of the interaction was investigated by varying the size of the supercell. The edge-to-edge distance between bubbles decreases with the size of the supercell because of the periodic replicas. Different sizes of supercell from $(9.8 \mathrm{~nm})^{3}$ to $(48.9 \mathrm{~nm})^{3}$ were thus tested for a $d_{0}=6.5 \mathrm{~nm}$ bubble at $300 \mathrm{~K}$, yielding an initial bubble separation from 3.3 to $42.4 \mathrm{~nm}$.

As seen in Fig. 9, the helium density is almost constant for distances larger than $16.5 \mathrm{~nm}$, confirming that interactions between bubbles are negligible for the largest considered supercell. An increase of the helium density by about

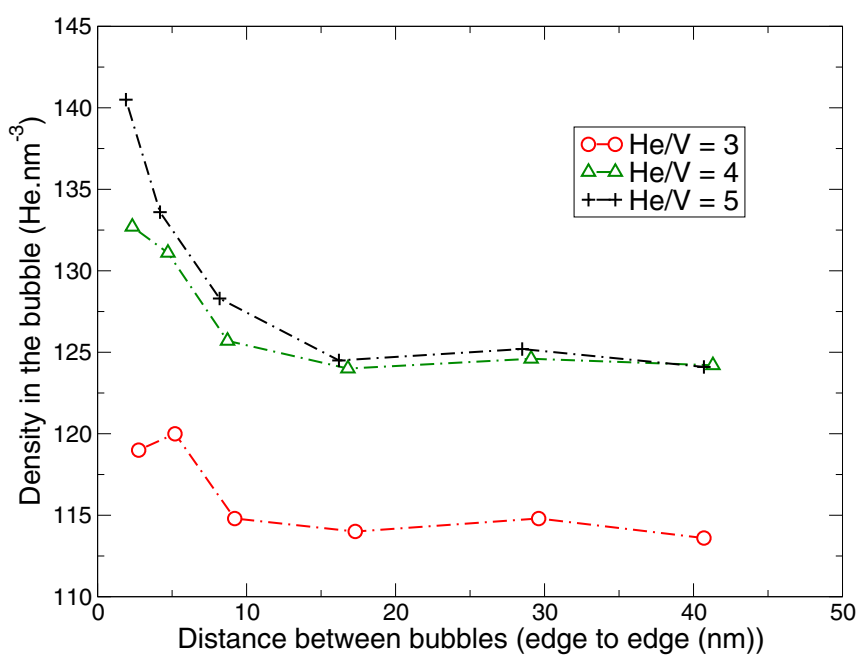

FIG. 9. Helium density vs distance between bubbles for different $\mathrm{He} / V$ ratios and for a bubble of initial diameter $d_{0}=6.5 \mathrm{~nm}$ at $300 \mathrm{~K}$.

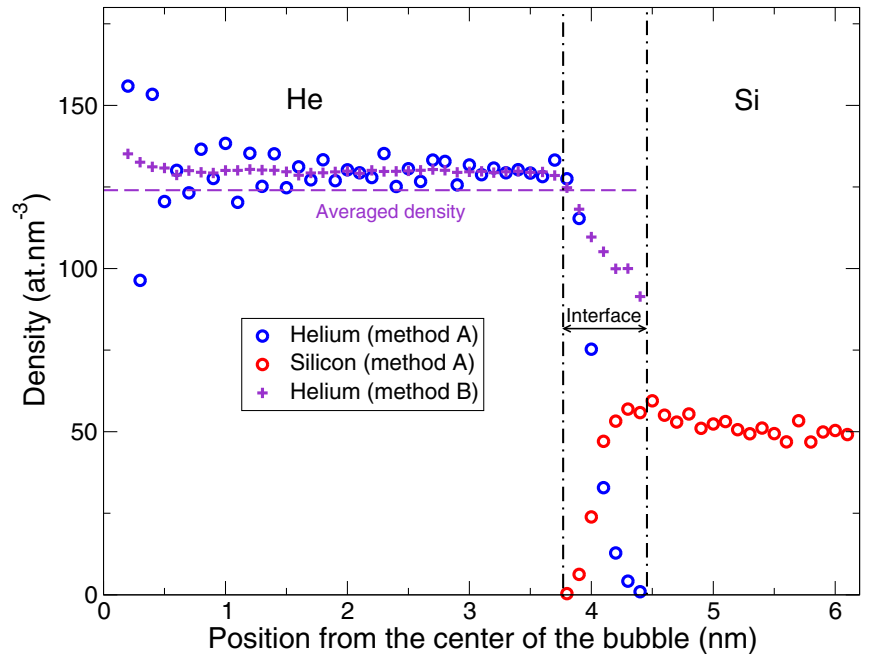

FIG. 10. Local density of helium and silicon as a function of the position from the center of the bubble. Blue and red symbols represent helium and silicon densities, respectively, obtained as the number of atoms in a spherical shell divided by its volume. The purple crosses correspond to the local helium density calculated using Voronoi volumes. The dashed line marks the averaged density determined with all helium atoms in the bubble. The initial bubble measured $6.5 \mathrm{~nm}$ in diameter filled with $\mathrm{He} / V=5(300 \mathrm{~K})$.

$10-15 \mathrm{He} \mathrm{nm}^{-3}$ is visible for a separation between bubbles of 2-5 nm, particularly for high $\mathrm{He} / V$ ratios; this result indicates that the interaction between bubbles confined in a band could lead to a helium density increase.

\section{Bubble-matrix interface}

In this section, we investigate the possible influence of the $\mathrm{Si}-\mathrm{He}$ interface in the calculation of the density. As previously discussed, a gap between helium and silicon atoms appears for a low helium density in the bubble. For higher ratios, an interface is made up of both helium and silicon atoms. Figure 10 represents the local density of helium and silicon atoms versus the position from the center of the bubble. Each point is calculated in a $1-\AA$-thick spherical shell region for a bubble of initial diameter $d_{0}=6.5 \mathrm{~nm}$ at a $\mathrm{He} / V=5$ ratio and at $300 \mathrm{~K}$, according to two different methods. In the first one, labeled $A$, the density is simply the number of atoms in the shell divided by the volume of the shell (circles in Fig. 10). In the second method, labeled $B$, the density is the number of atoms in the shell divided by the sum of the Voronoi volumes of these atoms (crosses in Fig. 10).

Three regions can be distinguished in Fig. 10. The first one, from 0 to $3.8 \mathrm{~nm}$, contains only helium atoms and thus corresponds to the bubble. The second region, from 3.8 to $4.4 \mathrm{~nm}$, is the interface of the bubble, including both $\mathrm{Si}$ and He atoms. The third region, for a position greater than $4.4 \mathrm{~nm}$, corresponds to the silicon bulk. The silicon density near the interface is higher than the bulk one because of the pressure transmitted from the bubble to the matrix.

When calculating using method $B$, the helium density in the bubble is close to the one calculated using method $A$, except in the center where method $A$ leads to large dispersion due to the 
small amount of atoms. In the interface, the helium density decreases for both methods but the meaning is different. Indeed, with method $A$, it indicates that there are fewer helium atoms in the region considered. With method $B$, the decrease in the helium density at the interface means that helium atoms occupy a greater volume at the interface than in the bubble. This might be due to the higher strength of the $\mathrm{Si}-\mathrm{He}$ interaction compared to the He-He one. In previous sections, the helium density was determined by summing the Voronoi volumes of all helium atoms, the usual approach in the literature. It corresponds here to averaging local densities obtained with method $B$. The decrease of the local density at the interface would lead to an underestimation of the total density. In fact, experimentally, the helium density is measured in the center of the bubble to avoid any surface effects. The density calculated in the center of the bubble is higher compared to the averaged density (purple dashed line, Fig. 10). In our case, the helium density can reach $130 \mathrm{He} \mathrm{nm}^{-3}$ in the center, whereas it is $124 \mathrm{He} \mathrm{nm}^{-3}$ by averaging over all helium atoms. This effect is stronger with small bubbles because the interface over volume ratio is higher.

\section{Influence of interstitial atoms}

At high $\mathrm{He} / V$ ratios, the maximum density in the bubble is mostly related to the deformation of the surrounding matrix. Then, by strengthening the matrix, a higher helium density in the bubble could be expected. One way to do so could be through the presence of helium atoms in the matrix. Note that this is not an unrealistic hypothesis because not all implanted helium atoms are necessarily located inside the bubbles. Here we consider single interstitial helium atoms, since it has recently been shown that helium clustering is not favored in silicon [63]. To estimate this effect on the final density, we investigated a $d_{0}=6.5 \mathrm{~nm}$ bubble in a $(9.8 \mathrm{~nm})^{3}$ supercell in order to include an interaction between bubbles. Helium interstitial atoms were added in a spherical shell of $0.8 \mathrm{~nm}$ width around the bubble, from $0 \%$ to $100 \%$ of the tetrahedral interstitial sites. Figure 11 shows the helium density, determined in the bubble center, as a function of the number of interstitial helium atoms. As assumed, adding interstitial atoms around the bubble delays the plastic deformation, thus increasing the maximum helium density. However, the effect remains quite small, even for a nonrealistic $100 \%$ interstitial configuration.

Finally, with a small box (interaction between bubbles), computing the density in the bubble center and with a reasonable proportion of $10 \%$ of helium interstitials, the helium density reaches $141 \mathrm{He} \mathrm{nm}^{-3}$, which is closer to the densities found experimentally after a $500^{\circ} \mathrm{C}$ annealing. Without considering these effects, the maximum helium density was about $125 \mathrm{He} \mathrm{nm}^{-3}$ at $300 \mathrm{~K}$.

\section{E. Influence of the interatomic potential}

Obviously, the strength of the matrix controls the final helium density in the bubble. The choice of the Si-Si potential might thus be important. Furthermore, our experiments unexpectedly revealed very high helium densities in the bubbles. In Ref. [51], it is mentioned that the MEAM He-He potential is

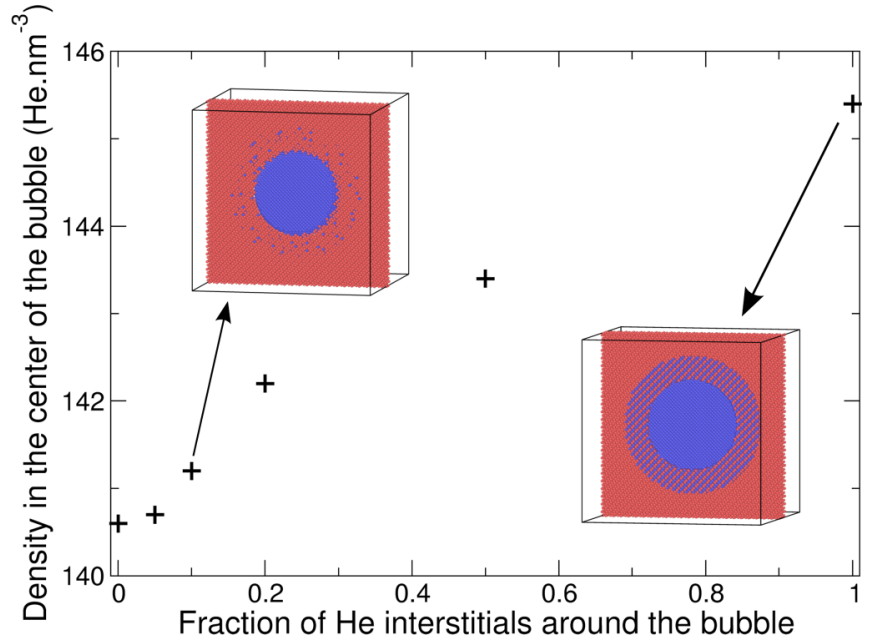

FIG. 11. Helium density in the center of the bubble vs the number of interstitial He atoms in the matrix for a $d_{0}=6.5 \mathrm{~nm}$ initial diameter bubble in a $(9.8 \mathrm{~nm})^{3}$ supercell, and a $\mathrm{He} / V=5$ ratio at $300 \mathrm{~K}$. Two snapshots are presented to show the interstitial atom distribution.

suited for pressure lower than $10 \mathrm{GPa}$ [51], corresponding to helium densities of about $135 \mathrm{He} \mathrm{nm}^{-3}$ [70]. It is therefore highly advisable to study the influence of the interatomic potential used in the calculations, as is done now.

To compare with the previously applied MEAM potential (denoted as MEAM-1 in the following), three additional potentials were tested. For all three, the Si-He interaction is modeled by a tabulated pair function extracted from a $\mathrm{SiC}-\mathrm{He}$ potential, which will be reported elsewhere, and the Beck potential [71] is used for the He-He part. This potential is particularly suited to describe helium at very high pressure. Different Si-Si interactions have been considered: Tersoff [72], Stillinger-Weber (SW) [73], and MEAM [60] (denoted as MEAM-2 in the following).

In all cases, we found that a maximum density value can be reached due to the plastic deformation of the silicon matrix by amorphization at the bubble-matrix interface. The fact that all potentials lead to similar behaviors gives us confidence in the robustness of our results. Figure 12 represents the final helium density value reached in a helium bubble, for different simulation conditions. In the case of an isolated bubble, a slightly higher value is obtained using SW compared to MEAM-1. However, noticeably larger densities are obtained using Tersoff and MEAM-2. Comparing MEAM-1 and MEAM-2 suggests that this change is essentially related to the different $\mathrm{He}-\mathrm{He}$ and $\mathrm{Si}-\mathrm{He}$ interactions. As can be seen, taking into account the presence of interactions between bubbles and helium atoms around bubbles leads to higher helium density regardless of the interatomic potential used. This confirms the general character of the effects investigated in this paper. The final densities for all potentials except MEAM-1 are in close agreement, in the range of 164-166 $\mathrm{He} \mathrm{nm}^{-3}$. Such values are now in agreement with experimental densities measured in samples annealed at $500^{\circ} \mathrm{C}$. This confirms that MEAM- 1 is not the most appropriate potential for modeling the helium bubbles measured in the latter case, since this potential has been designed for moderate density values lower than $135 \mathrm{He} \mathrm{nm}^{-3}$ [51]. 


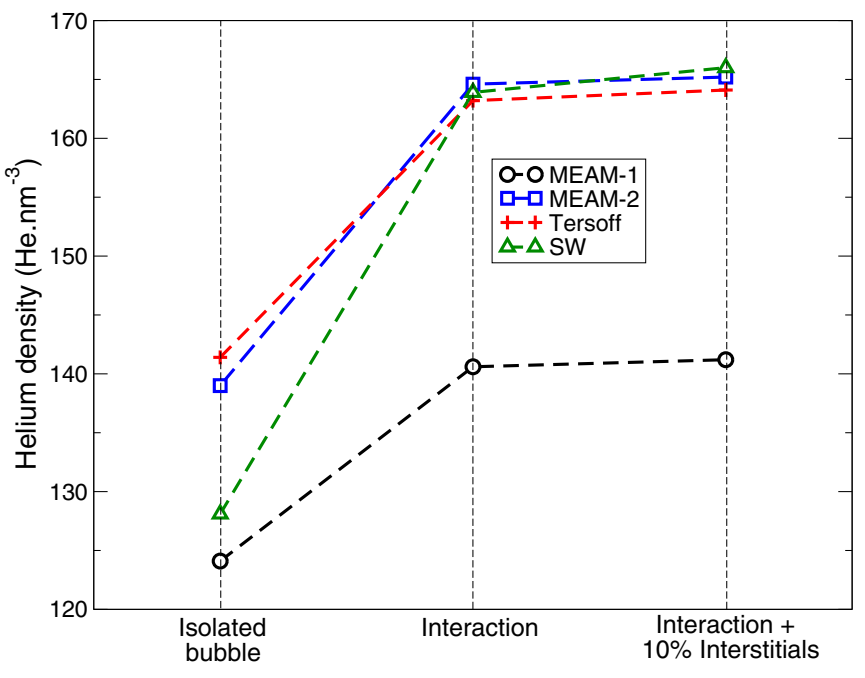

FIG. 12. Helium density (calculated in the bubble center) vs different simulation conditions calculated in an initial diameter $d_{0}=6.5 \mathrm{~nm}$ bubble at $300 \mathrm{~K}$, and an initial density $\mathrm{He} / V=5$. Different potentials are compared (see the text for explanations).

\section{DISCUSSION}

By considering only isolated bubbles, we found excellent agreement between simulations and measurements for the $700{ }^{\circ} \mathrm{C}$ annealed sample. However, it was initially not possible to reach the helium densities measured in the $500^{\circ} \mathrm{C}$ annealed sample. To understand this issue, several effects have been investigated and have been shown to influence the helium density determined by MD simulations.

In particular, we have shown that the interatomic potential plays an important role in the maximum calculated helium density, identifying almost $20 \mathrm{He} \mathrm{nm}^{-3}$ differences depending on the chosen potential. Nevertheless, by itself this effect is not sufficient to explain the differences with experimental data. However, when taking into account factors such as interaction between neighboring bubbles (leading to 4-13\% higher helium densities), the presence of helium interstitials ( $0.5 \%$ higher helium densities), and computation of the density in the center of the bubbles, better agreement is obtained. Considering these effects together, a density value of $165 \mathrm{He} \mathrm{nm}^{-3}$ is reached. Such a value is in the midrange of helium density measurements in the $500{ }^{\circ} \mathrm{C}$ annealed sample, suggesting that we have achieved good agreement between experiments and simulations. Particularly notable is the effect of interaction between bubbles. Previous investigations only considered isolated bubbles $[50,53,74]$. However, experimental observations clearly show that separations between bubbles can be just a few nanometers [39], which leads to a higher $\mathrm{He}$ density in the bubbles, likely because of a local strengthening of the matrix. Note that in a nuclear context bubbles can be isolated, thus with a lower bubble-bubble interaction.

Furthermore, other effects have been investigated with no noticeable effect on the helium density. In particular, we have observed that the way the helium atoms are inserted, as an hcp crystal or in random positions, leads to a negligible difference. Moreover, simulations in the $N V T$ or in the $N P T$ ensembles lead to similar results. Finally, as the bubbles created by the
$500{ }^{\circ} \mathrm{C}$ annealing exhibit an aspect ratio higher than 1 , we have also investigated bubbles with an aspect ratio up to 2.2. Again, the differences with spherical bubbles of the same equivalent diameter were negligible.

An interesting aspect revealed by MD simulations concerns the bubble-matrix interface, whose characteristics change drastically depending on the helium density. The gap identified at a low ratio between helium and silicon atoms $(\mathrm{He} / V=1)$ has already been observed for helium bubbles in iron $[46,50,66]$. This gap is ascribed to the stronger matrix-He repulsion than the He-He one [50]. By increasing the number of helium atoms in the bubble, the closure of the gap is visible [see Fig. 6(b)]. At high ratios, the interface is made up of both silicon and helium atoms, over an extent of a few angstroms (Fig. 10). Such a mixing has already been observed for bubbles in tungsten [74], where the interface thickness was of the same order of magnitude as in the present study $(0.6 \mathrm{~nm})$. As seen in Fig. 10, the density at the interface is lower than in the center of the bubble. Consequently, the helium density has to be carefully determined to avoid an overestimation (gap) or an underestimation (mixing), and only data from the center of the bubble should be taken into account.

It is customary and often more telling to discuss the bubble state in terms of internal pressure. So far, we only considered helium density since it is the direct outcome of experiments, while an equation of state (EOS) must be used to obtain the corresponding pressure. On the other hand, the internal pressure can be directly determined from virial stress calculations during MD simulations. Figure 13 shows the pressure in the bubbles as a function of the bubble diameter for both methods and using the EOS proposed by Trinkaus

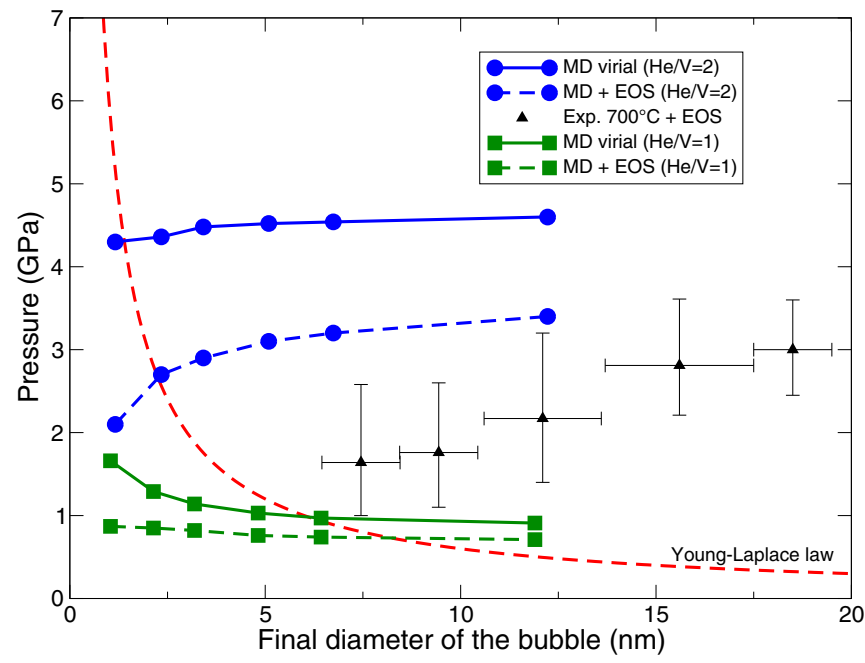

FIG. 13. Pressure vs final diameter of the bubble. Full lines correspond to the simulated bubbles, calculated from virial stress (filled symbols, full lines) and using the Trinkaus EOS (filled symbols, dashed lines). The black triangles correspond to the experimental densities converted in pressure using the Trinkaus EOS for the sample annealed at $700^{\circ} \mathrm{C}$. For the sake of clarity, an average of the experimental results was performed in five different regions, arbitrarily chosen. The error bars along the abscissa correspond to these regions, and the ordinate ones represent the standard deviation of the pressures in this region. 
[31]. These data are compared with the Young-Laplace law, using an average surface energy $\gamma=1.5 \mathrm{~J} \mathrm{~m}^{-2}$ [75]. Here, we have focused on the experimental data for the $700^{\circ} \mathrm{C}$ annealed sample and MD calculations with low initial He densities, both being in the same pressure range as this law. We first note that the pressures given by the EOS are typically lower than calculated pressures, suggesting that using a helium bulk EOS is probably not appropriate for bubbles as suggested by Caro et al. [50]. However, the same trends are observed in both cases, allowing for a qualitative comparison. In the case of experimental results, the pressure in the bubbles clearly increases with the diameter, in contradiction with the Young-Laplace law. The same trend is observed in simulations for a $\mathrm{He} / V=2$ ratio. Conversely, for the $\mathrm{He} / V=1$ ratio the pressure apparently decreases as a function of bubble size, although the variation is quite different from the YoungLaplace law. However, as explained previously, this is due to the calculation of the bubble volume and to the presence of a gap between the bubble and the matrix at low density. Our investigations, therefore, suggest that the Young-Laplace law cannot be satisfactorily used for describing bubbles formed in covalent materials in similar conditions. In fact, this law implies a mechanical equilibrium enforced by an unlimited supply of vacancies from the matrix. While such conditions are commonly found in metals, vacancies in covalent systems are usually scarce due to high formation energies, leading to nonequilibrated bubbles. This explains why we found much higher pressure in our bubbles compared to similar investigations in metals (0.1-2 GPa [35,36]).

Our simulations uncover that the helium density, and the corresponding pressure, are limited by the mechanical strength of the silicon matrix. Above a certain threshold, the bubble volume increases following the plastic deformation of the matrix by amorphization, which in turn leads to the saturation of the final helium density. Trinkaus described different mechanisms limiting the helium density [31], such as self-interstitial emission or dislocation loop punching. An analysis of the simulated systems did not reveal occurrences of the former process. Conversely, dislocation loop punching is observed, but only for the highest temperature MD simulations, and in limited amounts. In all cases, the main factor responsible for bubble expansion remains the amorphization of the matrix localized at the bubble-matrix interface. As far as we know, such a mechanism has not been previously suggested in the literature. An experimental confirmation is difficult, however, because the simulations show that the amorphized region is of limited extent around the bubbles.

\section{SUMMARY}

The properties of helium bubbles of nanometric size in silicon were investigated both experimentally and numerically. Measurements of the helium density in bubbles have been made using an EFTEM-SI methodology recently developed for this system [39] for a large number of single bubbles. For samples annealed at $500{ }^{\circ} \mathrm{C}$, we found a helium density in the range of $120-180 \mathrm{He} \mathrm{nm}^{-3}$ (bubbles of 7-16 $\mathrm{nm}$ in diameter), whereas samples annealed at $700{ }^{\circ} \mathrm{C}$ lead to lower helium densities, in the range of 40-90 $\mathrm{He} \mathrm{nm}^{-3}$ (bubbles of 6-20 nm in diameter). MD calculations were performed for bubbles of different diameters (1-13 nm), allowing us to make a direct comparison with experiments and to study small bubbles out of the reach of experiments. The calculations revealed that the maximum attainable helium density depends on the temperature and is critically related to the strength of the silicon matrix, which tends to yield by amorphization at the highest density levels. Helium densities were determined to range from 50 to $125 \mathrm{He} \mathrm{nm}^{-3}$, in excellent agreement with $700^{\circ} \mathrm{C}$ annealed samples. However, these values remain lower than densities measured in $500{ }^{\circ} \mathrm{C}$ annealed bubbles. Different effects that might change the helium density have thus been studied.

First, by tuning the supercell size, a bubble confinement effect has been revealed, leading to a higher density when the separation between bubbles is $2-3 \mathrm{~nm}$. This assumption is in agreement with our TEM observations of bubble distributions. Second, we have shown that the density should be calculated in the center of the bubble to avoid errors related to the bubble-matrix interface. Third, the influence of residual interstitial helium atoms in the vicinity of the bubble has been investigated. Albeit small, they induce a strengthening of the matrix leading to slightly higher densities. Finally, the influence of the chosen semiempirical interactions has been studied by considering alternative interatomic potentials. It is found that the use of a potential especially designed for high densities allows higher helium densities to be reached. Combining all these effects, a maximum helium density of $165 \mathrm{He} \mathrm{nm}{ }^{-3}$ is obtained, in excellent agreement with experiments made on $500{ }^{\circ} \mathrm{C}$ annealed samples.

To conclude, our investigations revealed that nanometricsized helium bubbles in silicon can exhibit very high helium densities, much higher than those usually encountered in metals and predicted by the Laplace-Young law. These results suggest that this law is not valid at this scale for such a covalent material. Our calculations also showed that the density threshold is essentially set by the plastic yielding of the matrix by local amorphization and the presence of a bubble confinement effect. To demonstrate the general character of these results, we plan to study helium bubbles in other covalent materials. For high strength materials such as silicon carbide or gallium nitride, one could then expect to reach very high helium densities. Experiments are in progress to confirm this idea.

\section{ACKNOWLEDGMENTS}

Computations were performed on the supercomputer facilities of the Mésocentre de Calcul de Poitou-Charentes. This work has been partially supported by the "Nouvelle Aquitaine" Region.
[1] Fundamental Aspects of Inert Gases in Solids, edited by S. E. Donnelly and J. H. Evans (Plenum Press, New York, 1991).
[2] V. Raineri, A. Battaglia, and E. Rimini, Gettering of metals by He induced voids in silicon, Nucl. Instrum. Methods Phys. Res. B 96, 249 (1995). 
[3] B. Holländer, S. T. Lenk, S. Mantl, H. Trinkaus, D. Kirch, M. Luysberg, T. Hakbarth, H.-J. Herzog, and P. F. P. Fichtner, Strain relaxation of pseudomorphic $\mathrm{Si}_{1-x} \mathrm{Ge}_{x} / \mathrm{Si}\left(\begin{array}{lll}1 & 0 & 0\end{array}\right)$ heterostructures after hydrogen or helium ion implantation for virtual substrate fabrication, Nucl. Instrum. Methods Phys. Res. B 175, 357 (2001).

[4] M. Bruel, Silicon on insulator material technology, Electron. Lett. 31, 1201 (1995).

[5] P. R. Heck, K. K. Marhas, P. Hoppe, R. Gallino, H. Baur, and R. Wieler, Presolar he and ne isotopes in single circumstellar $\mathrm{SiC}$ grains, Astrophys. J. 656, 1208 (2007).

[6] H. Trinkaus and B. N. Singh, Helium accumulation in metals during irradiation-Where do we stand? J. Nucl. Mater. 323, 229 (2003)

[7] S. E. Donnelly, The density and pressure of helium in bubbles in implanted metals: A critical review, Radiat. Eff. 90, 1 (1985).

[8] C. Templier, B. Boubeker, H. Garem, E. L. Mathé, and J. C. Desoyer, Precipitation of implanted xenon in silicon, Phys. Status Solidi A 92, 511 (1985).

[9] G. Faraci, A. R. Pennisi, and J.-L. Hazemann, XANES of high-pressure $\mathrm{Kr}$ clusters in Be and Si, Phys. Rev. B 56, 12553 (1997).

[10] R. S. Dhaka, C. Biswas, A. K. Shukla, S. R. Barman, and A. Chakrabarti, $\mathrm{Xe}$ and $\mathrm{Ar}$ nanobubbles in $\mathrm{Al}$ studied by photoemission spectroscopy, Phys. Rev. B 77, 104119 (2008).

[11] C. C. Griffioen, J. H. Evans, P. C. De Jong, and A. van Veen, Helium desorption/permeation from bubbles in silicon: A novel method of void production, Nucl. Instrum. Methods Phys. Res. B 27, 417 (1987).

[12] F. Corni, C. Nobili, G. Ottaviani, R. Tonini, G. Calzolari, G. F. Cerofolini, and G. Queirolo, Helium in silicon: Thermaldesorption investigation of bubble precursors, Phys. Rev. B 56, 7331 (1997).

[13] P. F. P. Fichtner, J. R. Kaschny, M. Behar, R. A. Yankov, A. Mücklich, and W. Skorupa, The effects of the annealing temperature on the formation of helium-filled structures in silicon, Nucl. Instrum. Methods Phys. Res. B 148, 329 (1999).

[14] G. F. Cerofolini, G. Calzolari, F. Corni, S. Frabboni, C. Nobili, G. Ottaviani, and R. Tonini, Thermal desorption spectra from cavities in helium-implanted silicon, Phys. Rev. B 61, 10183 (2000).

[15] E. Oliviero, M.-L. David, M.-F. Beaufort, J.-F. Barbot, and A. van Veen, On the effects of implantation temperature in helium implanted silicon, Appl. Phys. Lett. 81, 4201 (2002).

[16] M.-L. David, M.-F. Beaufort, and J.-F. Barbot, Effect of implant temperature on defects created using high fluence of helium in silicon, J. Appl. Phys. 93, 1438 (2003).

[17] S. Frabboni, F. Corni, C. Nobili, R. Tonini, and G. Ottaviani, Nanovoid formation in helium-implanted single-crystal silicon studied by in situ techniques, Phys. Rev. B 69, 165209 (2004).

[18] G. Faraci, A. R. Pennisi, F. Zontone, B. Li, and I. Petrov, Expansion and melting of Xe nanocrystals in Si, Phys. Rev. B 74, 235436 (2006).

[19] M.-F. Beaufort, J.-F. Barbot, M. Drouet, S. Peripolli, E. Oliviero, L. Amaral, and P. F. P. Fichtner, Nanocavities induced by neon plasma based ion implantation in silicon, Nucl. Instrum. Methods Phys. Res. B 257, 750 (2007).

[20] B. S. Li, C. H. Zhang, Y. T. Yang, L. Q. Zhang, and C. L. Xu, Microstructural evolution upon annealing in Ar-implanted Si, Appl. Surf. Sci. 257, 9183 (2011).
[21] K. Hojou and K. Izui, Bubbles in SiC crystals formed by helium ion irradiation at high temperatures, J. Nucl. Mater. 160, 147 (1988).

[22] J. Chen, P. Jung, and H. Trinkaus, Microstructural evolution of helium-implanted $\alpha$-SiC, Phys. Rev. B 61, 12923 (2000).

[23] P. Jung, H. Klein, and J. Chen, A comparison of defects in helium implanted $\alpha$ - and $\beta$-SiC, J. Nucl. Mater. 283-287, 806 (2000).

[24] E. Oliviero, M.-F. Beaufort, F. Pailloux, and J.-F. Barbot, Damage formation in high energy helium implanted $4 \mathrm{H}-\mathrm{SiC}$, Nucl. Instrum. Methods Phys. Res. B 218, 391 (2004).

[25] C. H. Zhang, Y. M. Sun, Y. Song, T. Shibayama, Y. F. Jin, and L. H. Zhou, Defect production in silicon carbide irradiated with $\mathrm{Ne}$ and Xe ions with energy of $2.3 \mathrm{MeV} / \mathrm{amu}$, Nucl. Instrum. Methods Phys. Res. B 256, 243 (2007).

[26] L. Vincent, T. Sauvage, G. Carlot, P. Garcia, G. Martin, M.-F. Barthe, and P. Desgardin, Thermal behavior of helium in silicon carbide: Influence of microstructure, Vacuum 83, S36 (2009).

[27] J.-F. Barbot, F. Pailloux, M.-L. David, L. Pizzagalli, E. Oliviero, and G. Lucas, Helium implanted gallium nitride evidence of gas-filled rod-shaped cavity formation along the c-axis, J. Appl. Phys. 104, 043526 (2008).

[28] M.-F. Beaufort, L. Pizzagalli, A. S. Gandy, E. Oliviero, D. Eyidi, and S. E. Donnelly, Solid-phase epitaxial regrowth of amorphous silicon containing helium bubbles, J. Appl. Phys. 104, 094905 (2008).

[29] E. Oliviero, M.-O. Ruault, B. Décamps, F. Fotuna, E. Ntsoenzok, O. Kaïtasov, and S. Collin, Synthesis of mesoporous amorphous silica by $\mathrm{Kr}$ and $\mathrm{Xe}$ ion implantation: Transmission electron microscopy study of induced nanostructures, Microporous Mesoporous Mater. 132, 163 (2010).

[30] A.-M. Seydoux-Guillaume, M.-L. David, K. Alix, L. Datas, and B. Bingen, Trapping of helium in nano-bubbles in euxenite: Positive identification and implications, Earth Planet. Sci. Lett. 448, 133 (2016).

[31] H. Trinkaus, Energetics and formation kinetics of helium bubbles in metals, Radiat. Eff. 78, 189 (1983).

[32] G. F. Cerofolini, F. Corni, S. Frabboni, C. Nobili, G. Ottaviani, and R. Tonini, Hydrogen and helium bubbles in silicon, Mater. Sci. Eng. Rep. 27, 1 (2000).

[33] A. Vom Felde, J. Fink, T. Müller-Heinzerling, J. Pflüger, B. Scheerer, G. Linker, and D. Kaletta, Pressure of Neon, Argon, and Xenon Bubbles in Aluminum, Phys. Rev. Lett. 53, 922 (1984).

[34] M. Maekawa and A. Kawasuso, Characterization of helium bubbles in Si by slow positron beam, J. Phys. Conf. Ser. 225, 012032 (2010).

[35] D. Taverna, M. Kociak, O. Stéphan, A. Fabre, E. Finot, B. Décamps, and C. Colliex, Probing Physical Properties of Confined Fluids within Individual Nanobubbles, Phys. Rev. Lett. 100, 035301 (2008).

[36] S. Fréchard, M. Walls, M. Kociak, J. P. Chevalier, J. Henry, and D. Gorse, Study by EELS of helium bubbles in a martensitic steel, J. Nucl. Mater. 393, 102 (2009).

[37] M.-L. David, F. Pailloux, V. Mauchamp, and L. Pizzagalli, In situ probing of helium desorption from individual nanobubbles under electron irradiation, Appl. Phys. Lett. 98, 171903 (2011).

[38] M.-L. David, K. Alix, F. Pailloux, V. Mauchamp, M. Couillard, G. A. Botton, and L. Pizzagalli, In situ controlled modification of the helium density in single helium-filled nanobubbles, J. Appl. Phys. 115, 123508 (2014). 
[39] K. Alix, M.-L. David, G. Lucas, D. T. L. Alexander, F. Pailloux, C. Hébert, and L. Pizzagalli, Gentle quantitative measurement of helium density in nanobubbles in silicon by spectrum imaging, Micron 77, 57 (2015).

[40] R. Schierholz, B. Lacroix, V. Godinho, J. Caballero-Hernández, M. Duchamp, and A. Fernández, STEM-EELS analysis reveals stable high-density $\mathrm{He}$ in nanopores of amorphous silicon coatings deposited by magnetron sputtering, Nanotechnology 26, 075703 (2015).

[41] P. Loubeyre, R. LeToullec, J. P. Pinceaux, H. K. Mao, J. Hu, and R. J. Hemley, Equation of State and Phase Diagram of Solid ${ }^{4}$ He from Single-Crystal X-Ray Diffraction Over a Large $P-T$ Domain, Phys. Rev. Lett. 71, 2272 (1993).

[42] C. A. Walsh, J. Yuan, and L. M. Brown, A procedure for measuring the helium density and pressure in nanometresized bubbles in irradiated materials using electron-energy-loss spectroscopy, Philos. Mag. A 80, 1507 (2000).

[43] K. Morishita, R. Sugano, B. D. Wirth, and T. Diaz de la Rubia, Thermal stability of helium-vacancy clusters in iron, Nucl. Instrum. Methods Phys. Res. B 202, 76 (2003).

[44] S. M. Valones, M. I. Baskes, and R. L. Martin, Atomistic model of helium bubbles in gallium-stabilized plutonium alloys, Phys. Rev. B 73, 214209 (2006).

[45] H.-Y. Wang, W.-J. Zhu, S.-J. Liu, Z.-F. Song, X.-L. Deng, X.-R. Chen, and H.-L. He, Atomistic simulations of the elastic properties of helium bubble embedded aluminum, Nucl. Instrum. Methods Phys. Res. B 267, 849 (2009).

[46] S. M. Hafez Haghighat, G. Lucas, and R. Schäublin, State of a pressurized helium bubble in iron, Europhys. Lett. 85, 60008 (2009).

[47] G. Lucas and R. Schäublin, Stability of helium bubbles in alphairon: A molecular dynamics study, J. Nucl. Mater. 386-388, 360 (2009).

[48] A. Couet, J.-P. Crocombette, and A. Chartier, Atomistic study of the thermodynamic equilibrium of nano-sized helium cavities in $\beta \mathrm{SiC}$, J. Nucl. Mater. 404, 50 (2010).

[49] Z. Bao-Ling, W. Jun, and H. Qing, Molecular dynamics study of helium bubble pressure in titanium, Chin. Phys. B 20, 036105 (2011).

[50] A. Caro, J. Hetherly, A. Stukowski, M. Caro, E. Martinez, S. Srivilliputhur, L. Zepeda-Ruiz, and M. Nastasi, Properties of helium bubbles in Fe and FeCr alloys, J. Nucl. Mater. 418, 261 (2011).

[51] L. Pizzagalli, M-L. David, and M. Bertolus, Molecular dynamics simulation of the initial stages of He bubbles formation in silicon, Modell. Simul. Mater. Sci. Eng. 21, 065002 (2013).

[52] B. L. Zhang, J. Wang, M. Li, and Q. Hou, A molecular dynamics study of helium bubble formation and gas release near titanium surfaces, J. Nucl. Mater. 438, 178 (2013).

[53] A. Abhishek, M. Warrier, R. Ganesh, and A. Caro, Growth and structural determination of He bubbles in iron/chromium alloys using molecular dynamics simulations, J. Nucl. Mater. 472, 82 (2016).

[54] J. F. Ziegler, SRIM: The stopping and range of ions in matter, http://www.srim.org.

[55] J. C. Rife, S. E. Donnelly, A. A. Lucas, J. M. Gilles, and J. J. Ritsko, Optical Absorption and Electron-Energy-Loss Spectra of Helium Microbubbles in Aluminum, Phys. Rev. Lett. 46, 1220 (1981).
[56] A. A. Lucas, J. P. Vigneron, S. E. Donnelly, and J. C. Rife, Theoretical interpretation of the vacuum ultraviolet reflectance of liquid helium and of the absorption spectra of helium microbubbles in aluminum, Phys. Rev. B 28, 2485 (1983).

[57] D. Babonneau, D. Lantiat, S. Camelio, J. Toudert, L. Simonot, F. Pailloux, M.-F. Denanot, and T. Girardeau, Gold and silver nanoparticles embedded in dielectric-capping layers studied by HAADF-STEM, Eur. Phys. J. Appl. Phys. 44, 3 (2008).

[58] S. Plimpton, Fast parallel algorithms for short-range molecular dynamics, J. Comput. Phys. 117, 1 (1995).

[59] M. I. Baskes, J. S. Nelson, and A. F. Wright, Semiempirical modified embedded-atom potentials for silicon and germanium, Phys. Rev. B 40, 6085 (1989).

[60] M. I. Baskes, Modified embedded-atom potentials for cubic materials and impurities, Phys. Rev. B 46, 2727 (1992).

[61] J. Rabier, L. Pizzagalli, and J.-L. Demenet, Dislocations in Silicon at High Stress, in Dislocation in Solids, edited by L. Kubin and J. P. Hirth (Elsevier, Amsterdam, 2010), Vol. 16, Chap. 93, p. 47.

[62] K. T. Tang and J. P. Toennies, The van der waals potentials between all the rare gas atoms from He to Rn, J. Chem. Phys. 118, 4976 (2003).

[63] L. Pizzagalli, M.-L. David, and A. Charaf-Eddin, Investigation of helium interstitials aggregation in silicon: Why bubbles formation by a self-trapping mechanism does not work, Nucl. Instrum. Methods Phys. Res. B 352, 152 (2015).

[64] E. Bitzek, P. Koskinen, F. Gähler, M. Moseler, and P. Gumbsch, Structural Relaxation Made Simple, Phys. Rev. Lett. 97, 170201 (2006).

[65] Y. Okada and Y. Tokumaru, Precise determination of lattice parameter and thermal expansion coefficient of silicon between 300 and 1500 K, J. Appl. Phys. 56, 314 (1984).

[66] T. Jourdan and J.-P. Crocombette, A variable-gap model for calculating free energies of helium bubbles in metals, J. Nucl. Mater. 418, 98 (2011).

[67] See Supplemental Material at http://link.aps.org/supplemental/ 10.1103/PhysRevB.96.014110 for a study of the deformation around the bubble.

[68] A. Stukowski and K. Albe, Extracting dislocations and nondislocation crystal defects from atomistic simulation data, Modell. Simul. Mater. Sci. Eng. 18, 085001 (2010).

[69] A. Stukowski, Visualization and analysis of atomistic simulation data with OVITO-The open visualization tool, Modell. Simul. Mater. Sci. Eng. 18, 015012 (2010).

[70] G. F. Cerofolini, G. Calzolari, F. Corni, C. Nobili, G. Ottaviani, and R. Tonini, Ultradense gas bubbles in hydrogen- or heliumimplanted (or coimplanted) silicon, Mater. Sci. Eng. B 71, 196 (2000).

[71] D. E. Beck, A new interatomic potential function for helium, Mol. Phys. 14, 311 (1968).

[72] J. Tersoff, Empirical interatomic potential for silicon with improved elastic properties, Phys. Rev. B 38, 9902 (1988).

[73] F. H. Stillinger and T. A. Weber, Computer simulation of local order in condensed phases of silicon, Phys. Rev. B 31, 5262 (1985).

[74] J. Cui, M. Li, J. Wang, and Q. Hou, Molecular dynamics study of helium bubble pressure in tungsten, Nucl. Instrum. Methods Phys. Res. Sect. B 352, 104 (2015).

[75] D. J. Eaglesham, A. E. White, L. C. Feldman, N. Moriya, and D. C. Jacobson, Equilibrium Shape of Si, Phys. Rev. Lett. 70, 1643 (1993). 